\title{
Generalized electro-biothermo-fluidic and dynamical modeling of cancer growth: state-feedback controlled cesium therapy approach
}

\author{
Murad Al-Shibli \\ College Requirement Unit, Abu Dhabi Polytechnic, Institute of Applied Technology, Abu Dhabi, United Arab Emirates. \\ Email: murad.alshibli@iat.ac.ae
}

Received 21 December 2010; revised 23 February 2011; accepted 5 July 2011.

\begin{abstract}
This paper develops a generalized dynamical model to describe the interactive dynamics between normal cells, tumor cells, immune cells, drug therapy, electromagnetic field of the human cells, extracellular heat and fluid transfer, and intercellular fractional mass of Oxygen, cell acidity and Pancreatin enzyme. The overall dynamics stability, controllability and observability have been investigated. Moreover, Cesium therapy is considered as a control input to the 11-dimensional dynamics using state-feedback controlled system and pole placement technique. This approach is found to be effective in driving the desired rate of tumor cell kill and converging the system to healthy equilibrium state. Furthermore, the ranges of the system dynamics parameters which lead to instability and growth of tumor cells have been identified. Finally, simulation results are demonstrated to verify the effectiveness of the applied approach which can be implemented successfully to cancer patients.
\end{abstract}

Keywords: Cancer; Tumor Growth; Tumor Dynamics and Modeling; Immune System; Cesium Therapy;

State-Feedback Control; Pole Placement

\section{INTRODUCTION}

There are over 200 different types of cancer that affect virtually every organ in the body. They can seem bewilderingly different but all cancers share certain features as outlined by Douglas Hanahan and Robert Weinberg [1]. Six essential alterations in cell physiology that collectively dictate malignant growth: self-sufficiency in growth signals, insensitivity to antigrowth signals, evasion of programmed cell death (apoptosis), limitless replicative potential, sustained angiogenesis, and tissue invasion and metastasi.
The technical report [2] presents what is known today concerning non-ionizing electromagnetic field interaction with the human body. Many significant and interesting effects are identified. As an example, both theories and observations link non-ionizing electromagnetic field to cancer in humans, in at least three different ways: as a cause, as a means of detection and as an effective treatment.

A phase-space analysis of a mathematical model of tumor growth with an immune response and chemotherapy is introduced in [3]. It is proved that all orbits and trajectories are bounded and converge to one of several possible equilibrium points. The addition of a drug to the system can move the solution trajectory into a desirable basin of attraction.

State Dependent Riccati Equation (SDRE) based optimal control technique to a nonlinear tumor growth model is applied in [4]. The model consists of three biological cells which are normal tissue, tumor and immune cells. The effect of chemotherapy treatment is also included in the model. Chemotherapy administration is considered as a control input to the nonlinear cancer dynamics and the amount of administered drug is determined by using SDRE optimal control. The optimal control is applied to the model in order not only to drive the tumor cells to the healthy equilibrium state but also to minimize the amount of the drug used.

A basic mathematical model of the immune response when cancer cells are recognized is proposed. The model consists of six ordinary differential equations [5]. It is extended by taking into account two types of immunotherapy: active immunotherapy and adoptive immunotherapy. An analysis of the corresponding models is made to answer the question which of the presented methods of immunotherapy is better.

Review [6] explains why mathematics is a powerful tool for interpreting such data by presenting case studies that illustrate the types of insight that realistic theoretical 
models of solid tumour growth may yield. These range from discriminating between competing hypotheses for the formation of collagenous capsules associated with benign tumours to predicting the most likely stimulus for protease production in early breast cancer.

Understanding the dynamics of human hosts and tumors is of critical importance [7]. A mathematical model was developed that explored the immune response to tumors that was used to study a special type of treatment. This treatment approach uses elements of the host to boost its immune response in the hopes that the host can clear the tumor.

Two models of optimizing tumor growth are presented in [8] In the first model optimal controls minimize the tumor volume for a given amount of angiogenic inhibitors to be administered while the second formulation tries to achieve a balance between tumor reduction and total amount of angiogenic inhibitors given. For both models a full synthesis of optimal solutions determined by portions of bang and singular controls with rest periods is presented. The differences in the two solutions are discussed.

A new mathematical model is developed for the dynamics between tumor cells, normal cells, immune cells, chemotherapy drug concentration and drug toxicity [9]. Then, the theorem of Lyapunov stability is applied to design treatment strategies for drug protocols that ensure a desired rate of tumor cell kill and push the system to the area with smaller tumor cells.

Cells, whether cancerous or normal can only live and reproduce (undergo mitosis) in a $\mathrm{pH}$ range of between 6.5 and 7.5. A healthy cell has a $\mathrm{pH}$ of 7.35 while a cancer cell is more acidic. When the $\mathrm{pH}$ of a cancer cell goes above 7.5 it dies and if it goes above 8.0 it will die in a matter of hours.

Every cell in the body works as milli-volt battery. To successfully bring nourishment in, and take poisons out, it has to be fully charged. In a cancerous cell, the cell voltage drops from 90 millivolts to less than 40 millivolts. When the cell voltage gets to the very bottom, only 5 substances can pass in or out of the cell. They are water, sugar, potassium, cesium and rubidium. Oxygen cannot enter into a cancer cell. Even if there is a lot of oxygen in the blood, it won't get into the cell.

Potassium ions are responsible for the ability of glucose to enter the cell. Potassium enters cancer cells in a normal manner so glucose still enters the cancer cell. Cancer cells have only $1 \%$ of the calcium content found in normal healthy cells. The calcium, magnesium and sodium ions, which are responsible for the intake of oxygen into the cell, cannot enter the cancer cell but the potassium ion still enters these cells. Thus we have cancer cells containing glucose but no oxygen.
A healthy individual has a blood oxygen level of between 98 and 100 as measured by a pulse oximeter. Nobel Prize Laureate, Dr. Otto Warburg, discovered that when he lowered the oxygen levels of tissues by $35 \%$ for 48 hours normal cells were converted into irreversible cancer cells [10]. Cancer patients have low levels of oxygen in their blood usually around 60 compared to normal values of about 100 by pulse oximetry. The common therapies used to treat cancer (chemotherapy and radiation) both cause drastic falls in the body's oxygen levels. Tissues that are acidotic contain low levels of oxygen whereas tissues that are alkalotic have high levels of oxygen.

When oxygen fails to enter the cell the cell's ability to control, its $\mathrm{pH}$ is lost and the cell becomes quite acidic. This is caused by the appearance of abnormal metabolism (anerobic glycolysis) in which glucose is converted (fermentation) into two particles of lactic acid. This production of lactic acid promptly lowers the ph within the cell to 6.5 or lower. The lactic acid damages the template for proper DNA formation. Messenger RNA is also changed so the ability of the cell to control its growth is lacking. Rapid and uncontrolled cancer cell growth and division occurs. Vitamin $\mathrm{C}$ and zinc are able to enhance the uptake of cesium, rubidium, and potassium into cancer cells.

Cancer cells develop a protein coating 13 times thicker than normal cells. This makes it difficult for the immune system to attack them. By ingesting high doses of pancreatin, you can actually dissolve cancer cells inside the body [11]. In the natural course of one's lifetime, millions of cancer cells develop, and are harmlessly digested by the immune system. The body uses pancreatin, a secretion from the pancreas to dissolve the cancer cells. As we age, the pancreas is less and less able to make this important substance. By taking pancreatin orally, it is possible to increase the levels of its active ingredients in the blood, thereby helping the body break down the cancer cells and remove them from circulation. The active ingredients in pancreatin which have shown to have tumor dissolving abilities are trypsin and chymotrypsin. These ingredients were taken out of virtually all the pancreatin supplements available to consumers years ago. These active ingredients are being bought in massive quantities by the sewerage industries to digest the sewerage into less noxious forms.

Although many improvements and mathematical modeling have been introduced in the treatment of cancer, but majority has been limited to of modeling of normal cells, tumor cells, immune cells and cancer therapy and toxicity effects. Moreover, development of treatment strategies requires many clinical experiments first on animals and then on humans in order to figure 
out a convenient way for the administration of the therapy. These experiments, in general, take a long time and most importantly may result in many deaths during the development period. Clinical experiments also reveal the fact that there is a strong relationship between the cancer state (the number and/or volume of tumor cells, tumor type), the immune system of the patient and the treatment strategy. Hence, understanding the dynamical behavior of cancer has received a great interest.

This paper develops a generalized dynamical model to describe the interactive dynamics between normal cells, tumor cells, immune cells, drug therapy, electromagnetic field of the human cell, extracellular heat and fluid transfer, and intercellular fractional mass of oxygen, cell acidity and pancreatin. The overall dynamics stability and controllability has been investigated. Moreover, Cesium therapy is considered as a control input to the 11-dimensional dynamics using state-feedback controlled system and pole placement technique. This approach is found to be effective in driving the desired rate of tumor cell kill and converging the system to healthy equilibrium state. Furthermore, the ranges of the system dynamics parameters which lead to instability and growth of tumor cells have been identified. Finally, simulation results are demonstrated to verify the effectiveness of the applied approach which can be implemented for each individual case.

This paper is organized as follows. Section 2 introduces modeling of the electromagnetic field of a live cell, cellular heat and fluid transfer are presented in Sections 3 and 4, respectively. Cellular factional composition is outlined in Section 5, generalized cellular-tumor dynamics is detailed in Section 6. Controllability and state-feedback control design of tumor dynamics are presented in Section 7. Section 8 demonstrates simulation which followed finally conclusions.

\section{ELECTROMAGNETIC CELLULAR MODELING}

A great variety of theories have been developed to describe the electro-magnetic field of the human body. Some theories regarded the human body as a whole as single prolate spheroid with a single set of electromagnetic constants: permittivity, permeability, and conductivity [2].

In that sense, the body is a simple antenna or probe capable of intercepting a certain amount of electromagnetic energy, which is converted entirely in to heat. At the other extreme, the body may be regarded as a collection of countless electronic microcircuits, each one corresponding to an individual cell or partially. Electromagnetic energy somehow finds its way to individual microcircuits and influences the electronic functions there. These functions include various communication and control processes essential to life and its activities. Efforts to understand these have focused attention on the microscopic components of the tissues such cells, membranes, fluids, molecules in solution rather than the tissue taken as a whole. Some theories have been developed concerning individual cells. An equivalent circuit has been developed as shown below. Given an incident current or current density actually passing across the cell membrane through and through the cell can be calculated. If the current is sufficiently enough, different responses are possible. For example a current density of 1 $\mathrm{mA} / \mathrm{cm}^{2}$ is about the amount associated with the action potential of nerve and muscle cells. Perhaps a pulsed electromagnetic filed could simulate these action potentials and confuse the body by generating false signals.

Of the cells part, the membrane probably has attracted most attention at least with respect top electromagnetic effects. Many membrane properties have been quantified. Typical thickness 45 Ang, typical capacitance 1 microfarad $/ \mathrm{cm}^{2}$, typical leakage conductance $1-10 \mathrm{mhos} / \mathrm{cm}^{2}$, typical resting potential 100 millivolts, dielectric constant 5, Electric field 22 Million V/m, Surface charge density $9.7 \times \mathrm{e}^{-8} \mathrm{C} / \mathrm{cm}^{2}=6.1 \times \mathrm{e}^{11}$ charge $/ \mathrm{cm}^{2}$. Of these perhaps the electric filed is the most remarkable one since it is almost greater than any other found in nature. For example the electric field of the Earth at its surface is only about $100 \mathrm{~V} / \mathrm{m}$.

So far the membrane has been treated as a homogenous substance and characterized by a capacitance and nonlinear conductance. In fact the membrane is not homogenous. The basic structure is a double layer of molecules called lipids. Lipids are hydrophobic, that is, they repel water. It is the repulsion that holds the membrane together. Proteins are chains of mino-acids. Proteins are so important because they can conduct charges while lipids are good insulators. Thus proteins are the conductance in the electronic circuit of the membrane.

Electromagnetic field can interact with proteins over a wide range of frequencies between 1 - $10 \mathrm{MHz}$. It depends on its size and mass protein can be modeled as single dipole which rotates in response to an oscillating field.

Proteins figure predominantly in at least one of the theories of cancer advanced by Nobel Laureate Albert Szent-Gyorgyi which currently being researched $[2,10]$. According to this theory, proteins conduct electron out of the cell interior. Oxygen molecules at the cell exterior accept the electrons and carry them away. These free electrons are the products of some chemical process inside the cell that inhibits reproduction. If the electrons are not conducted away, then the process stops, and the cell divides at uncontrollable rate. Eventually, there are 
enough cells to form a tumor which characterized by poorly circulated system. So, little or no oxygen-carrying blood reaches the cells.

The equivalent impedance (resistance) $Z_{e q}$ of the equivalent electric circuit of the human cell can now be expressed in the Laplace domain as:

$$
Z_{e q}=\frac{R_{i} R_{e} C_{w} s+2 R_{e}}{R_{i} R_{e} C_{w} s^{2}+\left(R_{i} C_{w}+R_{e} C_{w}+2 R_{e} C_{e}\right) s+2}
$$

where $R_{i}$ is the intracellular medium resistance, $R_{e}$ is the extracellular medium resistance, $C_{w}$ is the capacitance of the cell wall, $C_{e}$ is the capacitance of extracellular medium.

Now it is possible to express the voltage across the cell $V(s)$ using Ohm's law such that

$$
\frac{V(s)}{I(s)}=Z_{e q}
$$

where the $I(s)$ is the passing cellular current.

The voltage-current time change can be formulated by taking the Laplace inverse of the former equation as follows (assuming zero initial conditions)

$$
\begin{gathered}
R_{i} R_{e} C_{w} \ddot{V}(t)+\left(R_{i} C_{w}+R_{e} C_{w}+2 R_{e} C_{e}\right) \dot{V}(t)+2 V(t) \\
=R_{i} R_{e} C_{w} \dot{I}(t)+2 R_{e} I(t)
\end{gathered}
$$

The right hand side of the last Eq.3 represents the in put source to the cell. Since $R_{e}$ represents the extracellular resistance then the term $R_{e} I(t)=V_{e}(t)$ is the external voltage source to the cell and the term $R_{e} \dot{I}(t)=\dot{V}_{e x}(t)$ the rate of change of the external voltage source. The equation now can be modified to

$$
\begin{gathered}
R_{i} R_{e} C_{w} \ddot{V}(t)+\left(R_{i} C_{w}+R_{e} C_{w}+2 R_{e} C_{e}\right) \dot{V}(t)+2 V(t) \\
=R_{i} C_{w} \dot{V}_{e x}(t)+2 V_{e x}(t)=u_{e}(t)
\end{gathered}
$$
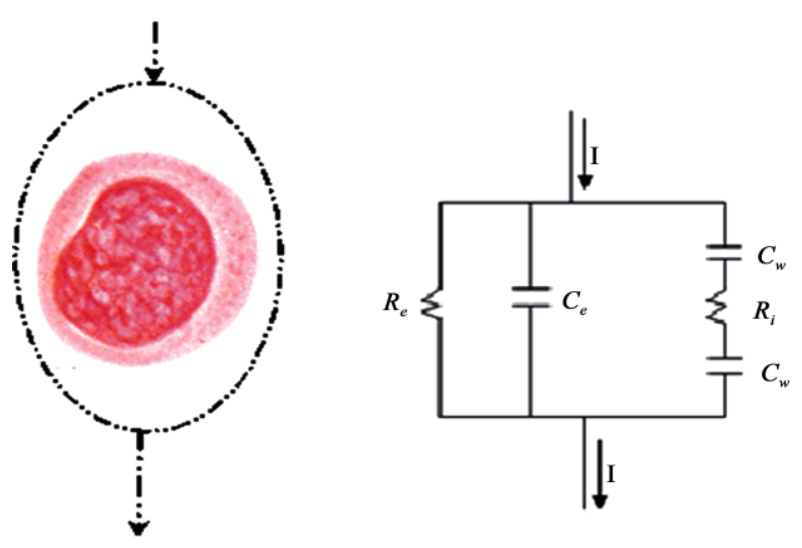

Figure 1. Passage of electric current $I$ in the human cell and its /equivalent electric circuit. $R_{e}$ is the extracellular resistance, $R_{i}$ is the intracellular resistance, $C_{w}$ is the capacitance of the wall of the cell and $C_{e}$ is the extracellular capacitance.
Eq.4 It represents an input-output relationship of the electric behavior of the cell in a second order ordinary linear differential equation form. Next section will introduce the dynamics of extracellular heat transfer as it is so important to maintain steady-state heat from and into the human cells.

\section{EXTRACELLULAR HEAT TRANSFER}

Thermal system involves transfer of heat from one cell to another. It may be analyzed I terms of thermal resistance and capacitance. To simplify the analysis it is assumed that the cellular thermal system can be represented by a lumped-parameter model, that substances that are characterized by resistance to heat flow have negligible heat capacitance, and that substances that are characterized by heat capacitance have negligible resistance to heat flow. Conduction and convection heat flow is only considered.

For conduction or convection heat transfer,

$$
q=K \Delta \theta
$$

where $q$ is the heat flow rate $(\mathrm{kcal} / \mathrm{sec}), \Delta \theta$ is the temperature difference $\left({ }^{\circ} \mathrm{C}\right)$ and $K$ is the heat coefficient $\left(\mathrm{kcal} / \mathrm{sec} .{ }^{\circ} \mathrm{C}\right)$. The coefficient $K$ is given by $K=k A / \Delta x$ for conduction and $K=H A$ for convection, where $k$ is thermal conductivity $\mathrm{kcal} / \mathrm{m} \cdot \mathrm{sec} .{ }^{\circ} \mathrm{C}$, $A$ is the area normal to heat flow $\mathrm{m}^{2}, \Delta x$ is the thickness of the conductor $(\mathrm{m})$ and $H$ is the convection coefficient $\left(\mathrm{kcal} / \mathrm{m}^{2} \cdot \mathrm{sec} .{ }^{\circ} \mathrm{C}\right)$.

Thermal resistance for heat transfer between two cells may be defined as

$$
R_{h}=\frac{\text { Change in Tepmerature Difference }}{\text { Change in Heat Flow Rate }}\left[\frac{{ }^{\circ} \mathrm{C}}{\mathrm{kcal} / \mathrm{sec}}\right]
$$

The thermal resistance for conduction or convection heat transfer is given by

$$
R_{h}=\frac{d(\Delta \theta)}{d q}=\frac{1}{K}
$$

The thermal capacitance

$$
C_{h}=\frac{\text { Change in Heat Stored }}{\text { Change in Temperature }}\left[\frac{k c a l}{{ }^{\circ} \mathrm{C}}\right]=m c_{h}
$$

where $m$ is mass of the cell considered $(\mathrm{kg})$, and $c$ is the specific heat of the cell $\mathrm{kcal} / \mathrm{kg} .{ }^{\circ} \mathrm{C}$.

It is desired now to conisder the tmprature change of a human cell such that fluid inside the cell is perfectly mixed such that the cell temprature is homogenoues. A single temprature is considred the intracellular temprature and the outflowing fluid.

The diffrential equation for the system is now

$$
C_{h} \frac{d \theta}{d t}=q_{\text {in }}-q_{\text {out }}
$$

where 


$$
q_{\text {out }}=\frac{1}{R}\left(\theta-\theta_{\text {out }}\right)
$$

Combing the last two Eqs.7-8 yields

$$
R_{h} C_{h} \dot{\theta}+\theta=R_{h} q_{\text {in }}+\theta_{\text {out }}
$$

It now possible now to express the generated cellular entropy $\dot{S}$ by $\dot{S}=\frac{\dot{q}}{\theta}$. Eq.9 shows that the temperature of the cell is changing as first order diffrentail dynamics given a heat input source and heat losses to the surrounding. Next section will consider how water flow in the cell changes.

\section{INTRACELLULAR FLUID MODELING}

Consider now the flow between two adjacent cells through their walls. The flow resistance for liquid flow through the cellular walls is defined as the change in the level difference necessary to cause a unit change in flow rate, that is

$$
R_{f}=\frac{\text { Change in Level Difference }}{\text { Change in Flow Rate }}\left[\frac{\mathrm{m}}{\mathrm{m}^{3} / \mathrm{sec}}\right]
$$

It is assumed that the flow is laminar. The relationship between the steady-state flow and steady-state head is defined as

$$
Q=K h
$$

where $Q$ is the steady-state flow $\operatorname{rate}\left(\mathrm{m}^{3} / \mathrm{sec}\right), K$ is flow coefficient $\left(\mathrm{m}^{2} / \mathrm{sec}\right)$, and $h$ is the steady-state head ( $\mathrm{m})$.

The capacitance $C$ of a tank is defined to be the change in quantity of stored fluid necessary to cause a unit change in the potential head.

$$
C_{f}=\frac{\text { Change in Lquid Stored }}{\text { Change in Head }}\left[\frac{\mathrm{m}^{3}}{\mathrm{~m}}\right]
$$

Since the inflow minus the outflow during a small time interval $d t$ is equal to the additional amount stored in the cell, then

$$
C_{f} d h=\left(Q_{\text {in }}-Q_{\text {out }}\right) d t
$$

From the definition of the flow resistance, the relationship between $Q_{\text {out }}$ and $h$ is given by

$$
Q_{\text {out }}=h / R_{h}
$$

The differential equation for the fluidic system now becomes

$$
R_{f} C_{f} \dot{h}+h=R_{f} Q_{i n}
$$

The former equation is a first order differential dynamical system show how the water content level is changing inside the cell given a regular water inflow.
Such a dynamics can be useful in modeling the fractional mass of substances inside the human cell. Oxygen, and hydrogen ions and Pancreatin are considered in this paper for their major role in keeping healthy cells and killing the tumor cells or at least stopping their growth.

\section{CELLULAR COMPOSITION DYNAMICS}

To determine the properties of a mixture, we need to know the composition of the mixture as well as the properties of the individual components. There are two ways to describe the composition of a mixture: either by specifying the number of moles of each component, called molar analysis, or by specifying the mass of each component, called gravimetric analysis.

Consider a fluid mixture composed of $n$ components. The mass of the mixture $m_{T}$ is the sum of the masses of the individual components, and the mole number of the mixture $N_{T}$ is the sum of the mole numbers of the individual components

$$
m_{T}=\sum_{1}^{n} m_{i} \text { and } N_{T}=\sum_{1}^{n} N_{i}
$$

The ratio of the mass of a component to the mass of the mixture is called the mass fraction $x_{i}$, and the ratio of the mole number of a component to the mole number of the mixture is called the mole fraction $y_{i}$ :

$$
x_{i}=\frac{m_{i}}{m_{T}} \text { and } y_{i}=\frac{m_{i}}{m_{T}}
$$

We can easily show that the sum of the mass fractions or mole fractions for a mixture is equal to 1

$$
\sum_{1}^{n} x_{i}=1 \text { and } \sum_{1}^{n} y_{i}=1
$$

It is assumed that the intercellular mixture is homogenous such that it is possible to express the he ratio of the mass of each substance with respect to the water volume as follows (substance-water ratio):

$$
v_{i}=\frac{m}{V}=\frac{m}{A h}
$$

where $v_{i}$ is the substance mass ration in a unit volume of water exists in the cell, mass $m$ of a substance, $V$ is the water volume, $A$ is the average cross sectional area of the cell, and $h$ is the water head indicator. The mass $m$ of a substance is related to the number of moles $N$ through the relation $m=N M$, where $M$ is the molar mass.

The former Eq.17 can now be modified to

$$
m=v_{i} A h
$$

The rate of change of each individual substance mass in the cellular fluid can be expressed as 


$$
\dot{m}=\dot{v}_{i} A h+v_{i} \dot{A} h+v_{i} A \dot{h}
$$

Next section will outline how this equation can be used to represent the contents of oxygen, hydrogen ions (as a measure of PH) and Pancreatin enzyme as a killer for the tumor cells.

\section{GENERALIZED \\ ELECTRO-BIO-THERMAL TUMOR DYNAMICS}

Mathematical models for cancer dynamics have been studied by many scientists using different mathematical methods. Some of these models consider the growth of tumor cells as population dynamics problems which include the interaction of tumor cells with other cells (e.g. normal cells and immune cells). In order to develop treatment strategies, the effects of therapy are also included in the models as control inputs. In this study, we analyze the model originally discussed in [4]. The model does not aim to concentrate on a specific kind of cancer and uses normalized parameters. It includes three different cell populations and chemotherapy drug concentration. Interaction of the tumor cells with normal and immune cell population in the absence of any treatment is given by the system

$$
\begin{aligned}
& \dot{x}_{1}=r_{1} x_{1}\left(1-b_{1} x_{1}\right)-c_{1} x_{1} x_{2} \\
& \dot{x}_{2}=r_{2} x_{2}\left(1-b_{2} x_{2}\right)-c_{2} x_{1} x_{2}-c_{3} x_{2} x_{3} \\
& \dot{x}_{3}=s_{0}+\rho \frac{x_{2} x_{3}}{\alpha+x_{2}}-c_{4} x_{2} x_{3}-d_{1} x_{3}
\end{aligned}
$$

where $x_{1}(t), x_{2}(t)$ and $x_{3}(t)$ denote the number of normal cells, the number of tumor cells and the number of immune cells at time $t$, respectively. The first term in the normal cell population is the logistic growth of normal cell population with growth rate $r_{1}$ and maximum carrying capacity $\left(1 / b_{1}\right)$. The second term is the loss of normal cells due to competition among tumor-normal cells for local resources. In a tumor cell population, the first term denotes the logistic growth of tumor cells in the absence of immune surveillance with the growth rate $r_{2}$ and maximum tumor carrying capacity $\left(1 / b_{2}\right)$.

The second and the third terms in (24) are death terms for tumor cells due to the interaction between immune and normal cells, respectively. Immune cells have a constant source $s_{0}$ which can be supplied from bone marrow or lymph nodes. In the presence of tumor cells, immune cells are stimulated by tumor cells with a Michaelis-Menten type saturation function with the positive parameters $\rho$ and $\alpha$. Immune cells are deactivated by tumor cells at the rate $c_{4}$ and they also die at the natural death rate $d_{1}$.

There are different ways to include the effect of chemotherapy in the tumor growth model. We assume that chemotherapy kills all cell populations with different ratios using mass action term. The effect of drug therapy in the model is shown with an additional state $x_{4}(t)$ and control input $u(t)$ which denote drug concentration in the blood stream, and external drug injection respectively. The nonlinear system (20)-(22) with the effect of drug therapy is:

$$
\begin{aligned}
& \dot{x}_{1}=r_{1} x_{1}\left(1-b_{1} x_{1}\right)-c_{1} x_{1} x_{2}-a_{1} x_{1} x_{4} \\
& \dot{x}_{2}=r_{2} x_{2}\left(1-b_{2} x_{2}\right)-c_{2} x_{1} x_{2}-c_{3} x_{2} x_{3}-a_{2} x_{2} x_{4} \\
& \dot{x}_{3}=s_{0}+\rho \frac{x_{2} x_{3}}{\alpha+x_{2}}-c_{4} x_{2} x_{3}-d_{1} x_{3}-a_{2} x_{3} x_{4} \\
& \dot{x}_{4}=-d_{2} x_{4}+u(t)
\end{aligned}
$$

Here, $a_{1}, a_{2}$, and $a_{3}$ are the different killing effects of chemotherapy on the cell populations. Chemotherapy drug decay rate in the blood stream is denoted by $d_{2}$. The system parameters which are normalized to the maximum carrying capacity of the normal cells are given in Table 1. Analysis of the model for possible equilibrium points in the absence of therapy is given in the next sub-section.

In order now to generalize the dynamics of the cell in terms of electromagnetic model, heat transfer model, fluid transfer model, fractional mass model, cellular growth, let us introduce the state space dynamic variables as follows.

Define the electric charge passing from or into the call $V(t)+$ as $x_{5}$. Since the electric current flow is the rate of change of charge passing through an inductor $I=\dot{q}$ then

$$
\begin{gathered}
\dot{x}_{5}=x_{6}=\dot{V}(t) \\
\dot{x}_{6}=\ddot{V}(t)
\end{gathered}
$$

Then the equivalent electric circuit Eq.4 can be reexpressed as

$$
\begin{gathered}
R_{i} R_{e} C_{w} \dot{x}_{6}(t)+\left(R_{i} C_{w}+R_{e} C_{w}+2 R_{e} C_{e}\right) x_{6}(t)+2 x_{5}(t) \\
=R_{i} C_{w} \dot{V}_{e x}(t)+2 V_{e x}(t)=u_{e}(t)
\end{gathered}
$$

The electric dynamics (29) can be modified as

$$
\begin{gathered}
\dot{x}_{6}=-\frac{\left(R_{i} C_{w}+R_{e} C_{w}+2 R_{e} C_{e}\right)}{R_{i} R_{e} C_{w}} x_{6} \\
-\frac{2}{R_{i} R_{e} C_{w}} x_{5}+u_{e}(t)
\end{gathered}
$$

Furthermore, representing the heat model (9) in the state space form assuming $x_{7}=\theta$ yields

$$
\dot{x}_{7}=-\frac{1}{R_{h} C_{h}} x_{7}+R_{h} q_{\text {in }}+\theta_{\text {out }}
$$


Table 1. Overall dynamics parameters.

\begin{tabular}{|c|c|}
\hline Parameter & Value \\
\hline$r_{1}$ & 1 \\
\hline$r_{2}$ & 1.5 \\
\hline$b_{1}$ & 1 \\
\hline$b_{2}$ & 1 \\
\hline$c_{1}$ & 1 \\
\hline$c_{2}$ & 0.5 \\
\hline$c_{3}$ & 1 \\
\hline$c_{4}$ & 1 \\
\hline$a_{1}$ & 1 \\
\hline$a_{2}$ & 0.15 \\
\hline$a_{3}$ & 0.05 \\
\hline$d_{1}$ & 0.2 \\
\hline$d_{2}$ & 1 \\
\hline$s_{0}$ & 0.33 \\
\hline$\rho$ & 0.1 \\
\hline$\alpha$ & 0.3 \\
\hline$R_{i}$ & $12 \times 10^{5}$ \\
\hline$R_{e}$ & $1 \times 10^{5}$ \\
\hline$C_{W}$ & $1 \times 10^{-6}$ \\
\hline$C_{e}$ & $1 \times 10^{-6}$ \\
\hline$R_{h}$ & $1 / 0.026$ \\
\hline$C_{h}$ & 1700 \\
\hline$R_{f}$ & $1 \times 10^{6}$ \\
\hline$C_{f}$ & 1600 \\
\hline$A$ & 1 \\
\hline$v_{\mathrm{O}_{2}}$ & 0.75 \\
\hline$v_{H^{+}}$ & 0.02 \\
\hline$v_{P n}$ & 0.05 \\
\hline$\rho_{A}$ & 1.9 \\
\hline$\rho_{O_{2}}$ & 0.21 \\
\hline$\rho_{H^{+}}$ & 0.01 \\
\hline$\rho_{P n}$ & 0.003 \\
\hline
\end{tabular}

Similarly, for the cellular fluid transfer (13) assuming $x_{8}=h \quad$ will lead to

$$
\dot{x}_{8}=-\frac{1}{R_{f} C_{f}} x_{8}+Q_{i n}
$$

For the significant of the oxygen, $\mathrm{pH}$ and Pancreatin enzyme for the survival of the normal cell, their fractional mass ratio will be considered. Denote their corresponding masses, respectively, as

$$
x_{9}=m_{\text {oxygene }}, \quad x_{10}=m_{H^{+}} \text {and } x_{11}=m_{\text {Pancreatin }}
$$

Referring to (18) and (19), last Eq.33 can be reformulated as

$$
\dot{m}_{i}=m_{i} \frac{\dot{v}_{i}}{v_{i}}+m_{i} \frac{\dot{A}}{A}+v_{i} A \dot{h}=\left(\rho_{v_{i}}+\rho_{A}\right) m_{i}+v_{i} A \dot{h}
$$

By the virtue of the fluid dynamics equation the former equation the fractional mass of the oxygen, $\mathrm{pH}$ and Pancreatin enzyme can be described, respectively, by

$$
\begin{gathered}
\dot{x}_{9}=-\frac{v_{O_{2}} A}{R_{f} C_{f}} x_{8}+\left(\rho_{O_{2}}+\rho_{A}\right) x_{9}+v_{O_{2}} A Q_{i n} \\
\dot{x}_{10}=-\frac{v_{H^{+}} A}{R_{f} C_{f}} x_{8}+\left(\rho_{H^{+}}+\rho_{A}\right) x_{10}+v_{H^{+}} A Q_{i n} \\
\dot{x}_{11}=-\frac{v_{P n} A}{R_{f} C_{f}} x_{8}+\left(\rho_{P n}+\rho_{A}\right) x_{11}+v_{P n} A Q_{i n}
\end{gathered}
$$

where $v_{\mathrm{O}_{2}}, v_{\mathrm{H}^{+}}$and $v_{\mathrm{Pn}}$ are the fractional ratios of oxygen, hydrogen as a measure of $\mathrm{PH}$ and Pancreatin, respectively. Fractional rate of change of the three substances with respect to their initial values are defined, respectively, as $\rho_{O_{2}}=\frac{\dot{v}_{O_{2}}}{v_{O_{2}}}, \quad \rho_{H^{+}}=\frac{\dot{v}_{H^{+}}}{v_{H^{+}}}$and $\rho_{P n}=\frac{\dot{v}_{p n}}{v_{P n}}$ along with the rate of change of the cell surface area with respect to its initial value as $\frac{\dot{A}}{A}=\rho_{A}$. Selection of such substances are based on their significant role in the survival of human cell and killing or at least slowing down the tumor cells.

Now the overall generalized model is

$$
\begin{aligned}
& \dot{x}_{1}=r_{1} x_{1}\left(1-b_{1} x_{1}\right)-c_{1} x_{1} x_{2}-a_{1} x_{1} x_{4} \\
& \dot{x}_{2}=r_{2} x_{2}\left(1-b_{2} x_{2}\right)-c_{2} x_{1} x_{2}-c_{3} x_{2} x_{3}-a_{2} x_{2} x_{4} \\
& \dot{x}_{3}=s_{0}+\rho \frac{x_{2} x_{3}}{\alpha+x_{2}}-c_{4} x_{2} x_{3}-d_{1} x_{3}-a_{2} x_{3} x_{4} \\
& \dot{x}_{4}=-d_{2} x_{4}+u(t) \\
& \dot{x}_{5}=x_{6} \\
& \dot{x}_{6}=-\frac{\left(R_{i} C_{w}+R_{e} C_{w}+2 R_{e} C_{e}\right)}{R_{i} R_{e} C_{w}} x_{6} \\
& -\frac{2}{R_{i} R_{e} C_{w}} x_{5}+u_{e}(t) \\
& \dot{x}_{7}=-\frac{1}{R_{h} C_{h}} x_{7}+R_{h} q_{\text {in }}+\theta_{\text {out }} \\
& \dot{x}_{8}=-\frac{1}{R_{f} C_{f}} x_{8}+Q_{i n} \\
& \dot{x}_{9}=-\frac{v_{O_{2}} A}{R_{f} C_{f}} x_{8}+\left(\rho_{O_{2}}+\rho_{A}\right) x_{9}+v_{O_{2}} A Q_{i n}
\end{aligned}
$$




$$
\begin{gathered}
\dot{x}_{10}=-\frac{v_{H^{+}} A}{R_{f} C_{f}} x_{8}+\left(\rho_{v, H^{+}}+\rho_{A}\right) x_{10}+v_{H^{+}} A Q_{i n} \\
\dot{x}_{11}=-\frac{v_{P n} A}{R_{f} C_{f}} x_{8}+\left(\rho_{v, P n}+\rho_{A}\right) x_{11}+v_{P n} A Q_{i n}
\end{gathered}
$$

The overall generalized dynamics represents the biothermal electro-fluidic dynamics with 11 dimension. Next section will investigate the stability of the entire dynamical system at equilibrium points.

\subsection{Equilibrium State}

It is necessary to investigate the equilibrium state of the cell since it indicates its stability and the non-growth of the tumor cells. As a start, let us assume a free therapy and find out the corresponding equilibrium points for the dynamics (38)-(41).

As for the normal cells, equilibrium is governed by $\dot{x}_{1}=r_{1} x_{1}\left(1-b_{1} x_{1}\right)-c_{1} x_{1} x_{2}=0$ indicates that $x_{1}=0, x_{2}=r_{1}\left(1-b_{1} x_{1}\right) / c_{1}$, and $x_{2}$ when $x_{1}=1 / b_{1}$

Tumor cells equilibrium exists when

$$
\dot{x}_{2}=r_{2} x_{2}\left(1-b_{2} x_{2}\right)-c_{2} x_{1} x_{2}-c_{3} x_{2} x_{3}=0
$$

at points satisfy

$$
x_{2}=0 \text { and } x_{2}=\left(1 / b_{2}\right)-\left(c_{2} / r_{2} b_{2}\right) x_{1}-\left(c_{3} /-r_{2} b_{2}\right) x_{3}
$$

On the other hand, the immune system will experience equilibrium when

$$
\dot{x}_{3}=s_{0}+\rho \frac{x_{2} x_{3}}{\alpha+x_{2}}-c_{4} x_{2} x_{3}-d_{1} x_{3}=0
$$

at state

$$
x_{3}=\frac{s_{0}\left(\alpha+x_{2}\right)}{d_{1}\left(\alpha+x_{2}\right)+c_{4} x_{2}\left(\alpha+x_{2}\right)-\rho x_{2}}
$$

Provided that $d_{1}\left(\alpha+x_{2}\right)+c_{4} x_{2}\left(\alpha+x_{2}\right) \neq \rho x_{2}$

Finally with free therapy $\dot{x}_{4}=\dot{0}$ at $x_{4}=0$ with zero control input $u(t)$.

It can be seen easily that the system has three different types of equilibria: Tumor-free (no tumor cells), Dead (no normal tissue cells), and Coexisting (both normal and tumor cells exist) equilibrium points [4]. In the context of developing therapy strategy, Tumor-free or Coexisting type equilibrium points should be reached, since in these types of states, the normal cell population is close to its healthy state. In this study, our aim is to determine the therapy dosage to bring the system to the tumor-free equilibrium point. The tumor-free equilibrium point of the system is obtained as $\left(1 / b_{2}, 0, s_{0} / d_{1}, 0\right)$ which gives us a healthy normal cell population of $\left(1 / b_{2}\right)$ and immune cell population of $\left(s_{0} / d_{1}\right)$ with zero tumor level and free drug injection. Considering now the elec- tromagnetic equilibrium given that $\dot{x}_{5}=x_{6}=0$, along with

$$
\dot{x}_{6}=-\frac{\left(R_{i} C_{w}+R_{e} C_{w}+2 R_{e} C_{e}\right)}{R_{i} R_{e} C_{w}} x_{6}-\frac{2}{R_{i} R_{e} C_{w}} x_{5}+u_{e}(t)=0
$$

when states $x_{6}=x_{5}=u_{e}(t)=0$ which is not feasible since the live cell should keep a cellular voltage in the range of 80 - 100 millivolts. With other equilibrium state

$$
x_{6}=-\frac{2}{R_{i} C_{w}+R_{e} C_{w}+2 R_{e} C_{e}} x_{5}+\frac{R_{i} R_{e} C_{w}}{R_{i} C_{w}+R_{e} C_{w}+2 R_{e} C_{e}} u(t)
$$

The human cell will have a steady-state heat transfer when

$$
\dot{x}_{7}=-\frac{1}{R_{h} C_{h}} x_{7}+R_{h} q_{\text {in }}+\theta_{\text {out }}=0
$$

at

$$
x_{7}=R_{h}^{2} C_{h} q_{\text {in }}+R_{h} C_{h} \theta_{\text {out }}
$$

A steady-state cellular fluid flow occurs when

$$
\begin{gathered}
\dot{x}_{8}=-\frac{1}{R_{f} C_{f}} x_{8}+Q_{i n}=0 \\
x_{8}=R_{f} C_{f} Q_{i n} .
\end{gathered}
$$

Thus, the fluid steady-state occurs when $x_{8}=R_{f} C_{f} Q_{i n}$. Fractional mass balance exist in the human cell when the oxygen flow, $\mathrm{PH}$ and Pancreatin satisfy, respectively,

$$
\begin{gathered}
\dot{x}_{9}=-\frac{v_{O_{2}} A}{R_{f} C_{f}} x_{8}+\left(\rho_{O_{2}}+\rho_{A}\right) x_{9}+v_{O_{2}} A Q_{i n}=0 \\
\dot{x}_{10}=-\frac{v_{H^{+}} A}{R_{f} C_{f}} x_{8}+\left(\rho_{v, H^{+}}+\rho_{A}\right) x_{10}+v_{H^{+}} A Q_{i n}=0 \\
\dot{x}_{11}=-\frac{v_{P n} A}{R_{f} C_{f}} x_{8}+\left(\rho_{v, P n}+\rho_{A}\right) x_{11}+v_{P n} A Q_{i n}=0
\end{gathered}
$$

with oxygen equilibrium state given by

$$
x_{9}=\frac{v_{O_{2}} A}{R_{f} C_{f}\left(\rho_{O_{2}}+\rho_{A}\right)} x_{8}-\frac{v_{O_{2}}}{\left(\rho_{O_{2}}+\rho_{A}\right)} A Q_{i n}
$$

and $\mathrm{PH}$ equilibrium state defined by

$$
x_{10}=\frac{v_{H^{+}} A}{R_{f} C_{f}\left(\rho_{v, H^{+}}+\rho_{A}\right)} x_{8}-\frac{v_{H^{+}}}{\left(\rho_{v, H^{+}}+\rho_{A}\right)} A Q_{i n}
$$

Finally Pancreatin stability when

$$
x_{11}=\frac{v_{P n} A}{R_{f} C_{f}\left(\rho_{v, P n}+\rho_{A}\right)} x_{8}-\frac{v_{P n}}{\left(\rho_{v, P n}+\rho_{A}\right)} A Q_{i n}
$$

Since the normal cell-tumor-immune dynamics is nonlinear, it is interesting to linearized the system at 
equlibruim points for the purposed of control design.

\subsection{Authors and Affiliations}

It is desired at the moment to seek a linearized model of the cells growth at the equilibrium points with zero control input in the given form

$$
\dot{\mathbf{x}}=\mathbf{A x}
$$

where $\mathbf{A}$ is the Jacobian Matrix. To fulfill this goal, let

$$
\begin{aligned}
& \dot{x}_{1}=f_{1}\left(x_{1}, x_{2}, x_{3}, x_{4}\right) \\
& \dot{x}_{2}=f_{1}\left(x_{1}, x_{2}, x_{3}, x_{4}\right) \\
& \dot{x}_{3}=f_{1}\left(x_{1}, x_{2}, x_{3}, x_{4}\right) \\
& \dot{x}_{4}=f_{1}\left(x_{1}, x_{2}, x_{3}, x_{4}\right)
\end{aligned}
$$

The Jacobian matrix for the dynamics (38)-(41) can be verified as

$$
A=\left[\begin{array}{cccc}
r_{1}-2 r_{1} b_{1} x_{1}-c_{1} x_{2} & -c_{1} x_{1} & 0 & -a_{1} x_{1} \\
-c_{2} x_{2} & r_{2}-2 r_{2} b_{2} x_{2}-c_{2} x_{1}-c_{3} x_{3}-a_{2} x_{4} & -c_{3} x_{2} & -a_{2} x_{2} \\
0 & \rho \frac{x_{3}\left(\alpha+x_{2}\right)-x_{2} x_{3}}{\left(\alpha+x_{2}\right)^{2}} & \rho \frac{x_{2}}{\alpha+x_{2}}-c_{4} x_{2}-a_{2} x_{4}-d_{1} & -a_{3} x_{3} \\
0 & 0 & 0 & -d_{1}
\end{array}\right]
$$

Substituting now the equilibrium point $\left(1 / b_{2}, 0, s_{0} / d_{1}, 0\right)$ yields to the following linearized Jacobia Matrix

$$
A=\left[\begin{array}{cccc}
r_{1}-2 r_{1} b_{1} / b_{2} & -c_{1} / b_{2} & 0 & -a_{1} / b_{2} \\
0 & r_{2}-c_{2} / b_{2}-c_{3} s_{0} / d_{1} & 0 & 0 \\
0 & \frac{s_{0} \rho}{d_{1} \alpha} & -d_{1} & -a_{3} s_{0} / d_{1} \\
0 & 0 & 0 & -d_{1}
\end{array}\right]
$$

The following tables show the generalized system parameters values used in the modeling and linearized state-feedback control.

This linearized overall dynamical model will be the interest of next section to investigate controllability, observability and designing a state-feedback controller using pole placement approach. Later analysis will show these parameters are so important in determining the stability of the human cell, its survival and in the growth of the tumor cells and decreasing the immune system. Some parameters affect the normal cells growth, tumor growth and immune system strength, cellular voltage, heat and fluid flow, oxygen rate, acidity and pancreatin enzyme.

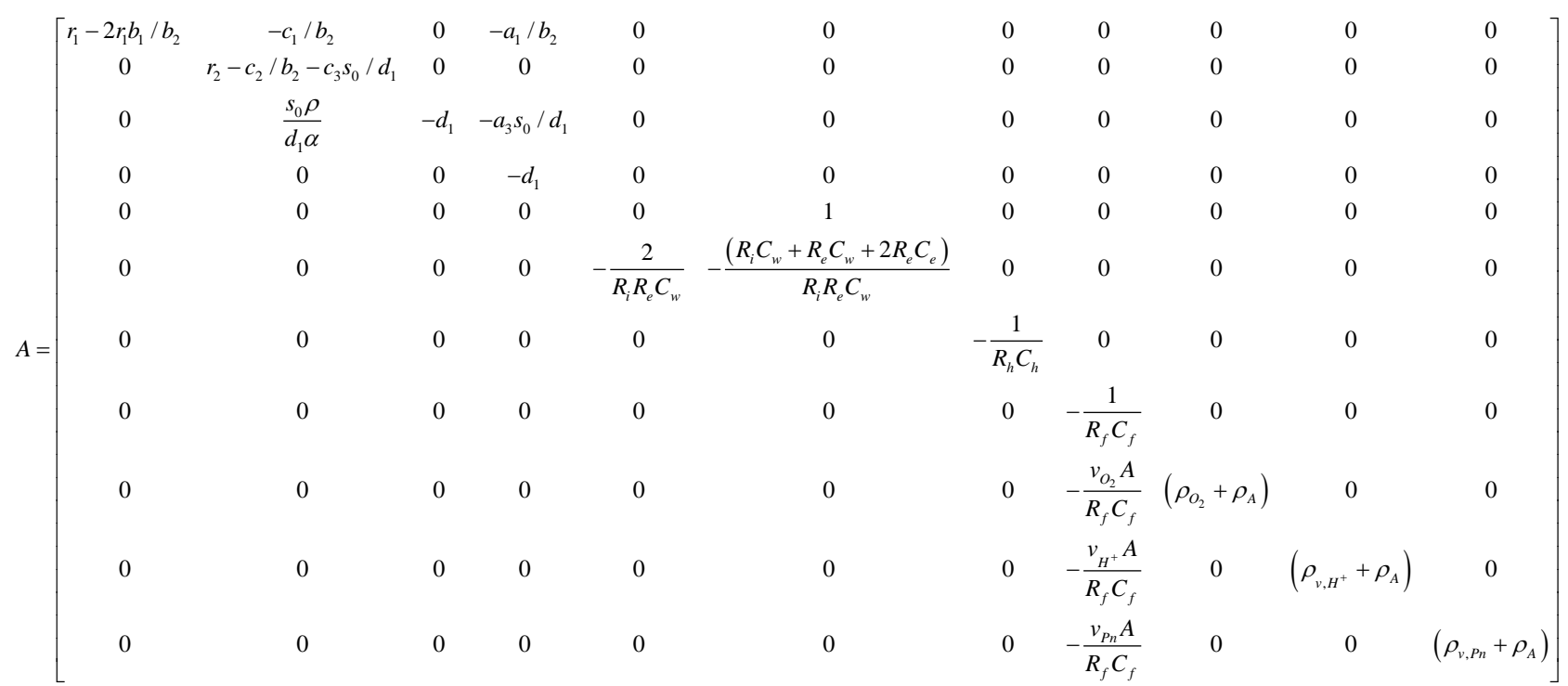

The input vector is given by

$$
B^{T}=\left[\begin{array}{lllllllllll}
1 & 1 & 1 & u(t) & 1 & u_{e}(t) & R_{h} q_{\text {in }}+\theta_{\text {out }} & Q_{\text {in }} & v_{O_{2}} A Q_{i n} & v_{H^{+}} A Q_{i n} & v_{P n} A Q_{\text {in }}
\end{array}\right]
$$




\section{CONTROLABILITY AND OF TUMOR DYNAMICS AND CONTROLLER DESIGN}

A system is said to be controllable at time $t_{0}$ if it is possible by means of unconstrained control vector to transfer the system from an initial state $x\left(t_{0}\right)$ to any other state in a finite interval time. The concepts of controllability and observability were introduced by Kalman. They play an important role in the design of control systems in state space. In fact, the conditions of controllability and observability may govern the existence of a complete solution to the control system design. Although most physical systems are controllable and observable, corresponding mathematical models may not possess the property of controllability and observability. In what follows, we shall derive the condition for complete state controllability. Figures $\mathbf{2}$ and $\mathbf{3}$ show the open-loop cancer controlled systems and closed-loop cancer controlled system, respectively.

Consider the continuous-time system

$$
\begin{array}{r}
\dot{\mathbf{x}}=\mathbf{A x}+\mathbf{B} w \\
\mathbf{y}=\mathbf{C x}+\mathbf{D} w
\end{array}
$$

where,

$$
\begin{aligned}
& \mathbf{x} \text { is a state vector } \\
& \mathbf{y} \text { is } m \text {-output vector } \\
& w \text { is a control signal } \\
& \mathbf{A} \text { is } n \times n \text { matrix } \\
& \mathbf{B} \text { is } n \times 1 \text { matrix } \\
& \mathbf{C} \text { is } n \times m \text { matrix }
\end{aligned}
$$

The system described in Eq.73 is said to be state controllable at $t=t_{0}$ if it is possible to construct an unconstrained control signal that will transfer an initial state to any final state in a finite time interval $t_{0} \leq t \leq t_{1}$. If every state is controllable, then the system is said to be completely state controllable [12]. The system is said to be controllable if and only if the following $n \times n$ matrix is full rank $n$

$$
\left[\begin{array}{lllll}
\mathbf{B} & \mathbf{A B} & \mathbf{A}^{2} \mathbf{B} & \cdots & \mathbf{A}^{\mathbf{n}-1} \mathbf{B}
\end{array}\right]
$$

This matrix is called the controllability matrix.

A system is said to be observable at time $t_{0}$, if with the system in state $x\left(t_{0}\right)$, it is possible to determine its state from the observation of the output over a finite time interval.

The concept of observability is very important because, in practice, the difficulty is encountered with state feedback control is that some of the state variables are not accessible for direct measurement, with the result that it becomes necessary to estimate the unmeasurable state variables in order to construct the control signals. The system is said to be observable if and only if the following $n \times n m$ matrix is of full rank $n$

$$
\left[\begin{array}{lllll}
\mathbf{C}^{T} & \mathbf{A}^{T} \mathbf{C}^{T} & \left(\mathbf{A}^{T}\right)^{2} \mathbf{C}^{T} & \cdots & \left(\mathbf{A}^{T}\right)^{\mathbf{n}-\mathbf{1}} \mathbf{C}^{T}
\end{array}\right]
$$

Matrix (76) is commonly called observability matrix.

This following analysis presents a design method commonly called the pole-placement technique. We assume that all state variables are measureable and are available for feedback. It is shown that if the system considered is completely state controllable, then poles of the closed-loop system may be placed at any desired locations by means of state feedback through an appropriate state feedback gain matrix. Let us assume the desired closed-poles are to be at $s_{1}=\mu_{1}, s_{2}=\mu_{2}, \ldots$, $s_{n}=\mu_{n}$.

We shall choose the control signal to be

$$
w=-\mathbf{K} \mathbf{x}
$$

This means that the control signal is determined by an instantaneous state. Such a scheme is called state feedback. The $1 \times n$ matrix $\mathbf{K}$ is called the state feedback gain matrix. Substituting (77) into Eq.73 gives

$$
\dot{\mathbf{x}}(t)=(\mathbf{A}-\mathbf{B K}) \mathbf{x}(t)
$$

The solution of this equation is give by

$$
\mathbf{x}(t)=\mathbf{x}(0) e^{(\mathbf{A}-\mathbf{B K}) t}
$$

where is the initial state caused by external disturbances. The stability and transient response characteristics are determined by the egienvalues of matrix $\mathbf{A}-\mathbf{B K}$. If matrix $\mathbf{K}$ is chosen properly, the matrix $\mathbf{A}-\mathbf{B K}$ can be made asymptotically stable matrix.

Define a transformation matrix $\mathbf{T}$ by

$$
\mathbf{T}=\mathbf{M W}
$$

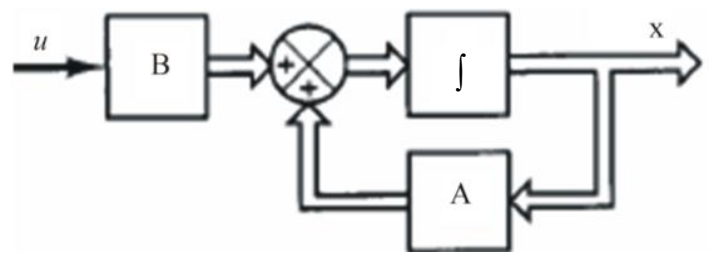

Figure 2. Open-loop cancer controlled system.

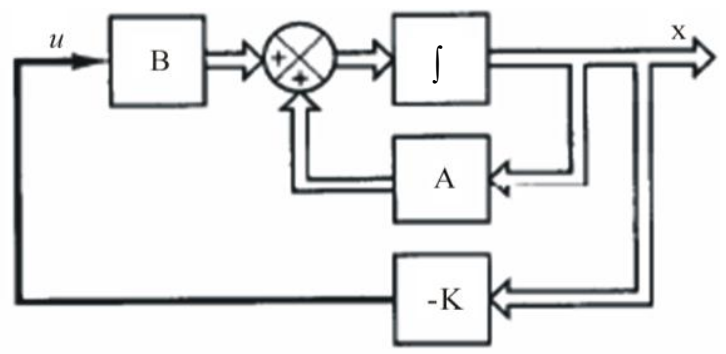

Figure 3. Closed-loop cancer controlled system. 
where $\mathbf{M}$ is the controllability matrix

$$
\left[\begin{array}{llll}
\mathbf{B} & \mathbf{A B} & \mathbf{A}^{2} \mathbf{B} & \mathbf{A}^{\mathbf{n}-\mathbf{1}} \mathbf{B}
\end{array}\right]
$$

and

$$
\mathbf{W}=\left[\begin{array}{ccccc}
a_{n-1} & a_{n-2} & \cdots & a & 1 \\
a_{n-2} & a_{n-3} & \vdots & 1 & 0 \\
\vdots & \vdots & \vdots & \vdots & \vdots \\
a_{1} & 1 & \cdots & 0 & 0 \\
1 & 0 & \cdots & 0 & 0
\end{array}\right]
$$

where the $a_{i}^{\prime} s$ are the coefficients of the characteristic polynomial

$$
|s \mathbf{I}-\mathbf{A}|=s^{n}+a_{1} s^{n-1}+\cdots+a_{n-1} s+a_{n}
$$

Let us choose a set desired egienvalues as $s_{1}=\mu_{1}$, $s_{2}=\mu_{2}, \cdots, s_{n}=\mu_{n}$. Then the desired characteristic equation becomes

$$
\left(s-\mu_{1}\right)\left(s-\mu_{2}\right) \cdots\left(s-\mu_{n}\right)=s^{n}+\alpha_{1} s^{n-1}+\cdots+\alpha_{n-1} s+\alpha_{n}
$$

The sufficient condition that the system to be completely controllable with all egienvalues arbitrarily placed by choosing the gain matrix

$$
\mathbf{K}=\left[\begin{array}{lllll}
\left(\alpha_{n}-a_{n}\right) & \left(\alpha_{n-1}-a_{n-1}\right) & \cdots & \left(\alpha_{2}-a_{2}\right) & \left(\alpha_{1}-a_{1}\right)
\end{array}\right] \mathbf{T}^{-1}
$$

The former outlined control design will be validated in the following section by demonstrating the simulation results.

\section{Simulation Results}

Every cell in the human body functions as a micro battery. To successfully bring nourishment in and take poisons out, it has to be fully charged. In a cancerous cell, the cell voltage drops from 90 millivolts to less than 40 millivolts. When the cell voltage gets to the very bottom, only 5 substances can pass in or out of the cell. They are water, sugar, potassium, cesium and rubidium [12]. Oxygen cannot enter into a cancer cell. Even if there is a lot of oxygen in the blood, it cannot get into the cell. Cesium, because of its electrical properties can still enter the cancerous cell. When it does so, because of its extreme alkalinity, the cell dies. Luckily, healthy cells are not affected by cesium because their cell voltage allows them to balance themselves. This uptake can be enhanced by Vitamins $\mathrm{A}$ and $\mathrm{C}$ as well as salts of zinc and selenium. The quantity of cesium taken up was sufficient to raise the cell to the $8 \mathrm{pH}$ range. Where cell mitosis ceases and the life of the cell is short.

The objective of the Cesium therapy is to kill the tumor cells, minimize the amount of drug application and to keep cellular voltage, thermo-fluid transfer and cellu- lar ingredients at fixed rates. In the simulated generalized cancer model, the healthy equilibrium point with the parameter set given in Table 1 is $(1,0,1.65,0,0.1,0$, $\left.7.4488 \times 10^{6}, 1.6 \times 10^{6}, 0,0,0\right)$ is locally stable. It means that the growth of cancer is controllable if sufficient drug surveillance is guaranteed.

In the absence of sufficient immunee control, the tumor cells grow in number and kill the healthy tissue cells and reach the limit capacity, which is referred to as dead equilibrium point. The initial states, i.e., the conditions when the chemotherapy treatment is started, are assumed to be: $N(0)=1, T(0)=0.20, I(0)=1, M(0)=1)$. The response of treatment-free cancer growth with respect to normalized time scale is given in Figures 4-6. Simulation analysis shows the following sensitivity of parameters that affect the cell stability.

The egienvalues of matrix A have some positive values, zero, and imaginary with negative real values. Thus the original system is unstable. These egienvalues are listed as follows:

$$
\begin{aligned}
& -0.0002+0.1291 \mathrm{i}, \\
& -0.0002-0.1291 \mathrm{i} \\
& -1.0000, \\
& -0.2000 \\
& -0.6500 \\
& -0.2000 \\
& -0.0000 \\
& 2.0050 \\
& 2.2100 \\
& 2.0100 \\
& -0.0000
\end{aligned}
$$

Since the original dynamics is unstable, it is now desired to design a state-feedback controller with the following desired egienvalues: $-0.1,-0.1,-0.2,-0.2,-0.3$, $-0.3,-0.4,-0.4,-0.5,-0.1,-0.1$. As it can be seen, all desired eigenvalues are negative and close to the origin to guarantee a stable and fast response.

Before proceeding in the controller design, the controllability condition defined by Eq.75 must be satisfied. Considering coefficient matrix A defined (71), the input vector given by (72), and parameters values listed in Table 1, it is found out that the controllability matrix is of full rank 11.

Simulation results in Figures 4-6 shows that the cesium therapy is so effective in brining the normal and immune system to its equilibrium state and forcing the tumor cell kill. As it can be seen from the earlier analayis, having no closed-loop controlled tumor therapy, the overall bio-thermo-fluidic-dynamical system was unstable. Enforcing a state-feedback pole placement cloride-cesium tumor therapy, a complete therapy of disease is achieved as shown in Figure 4. Normal and immune cells have been brought to an equilibrium state 

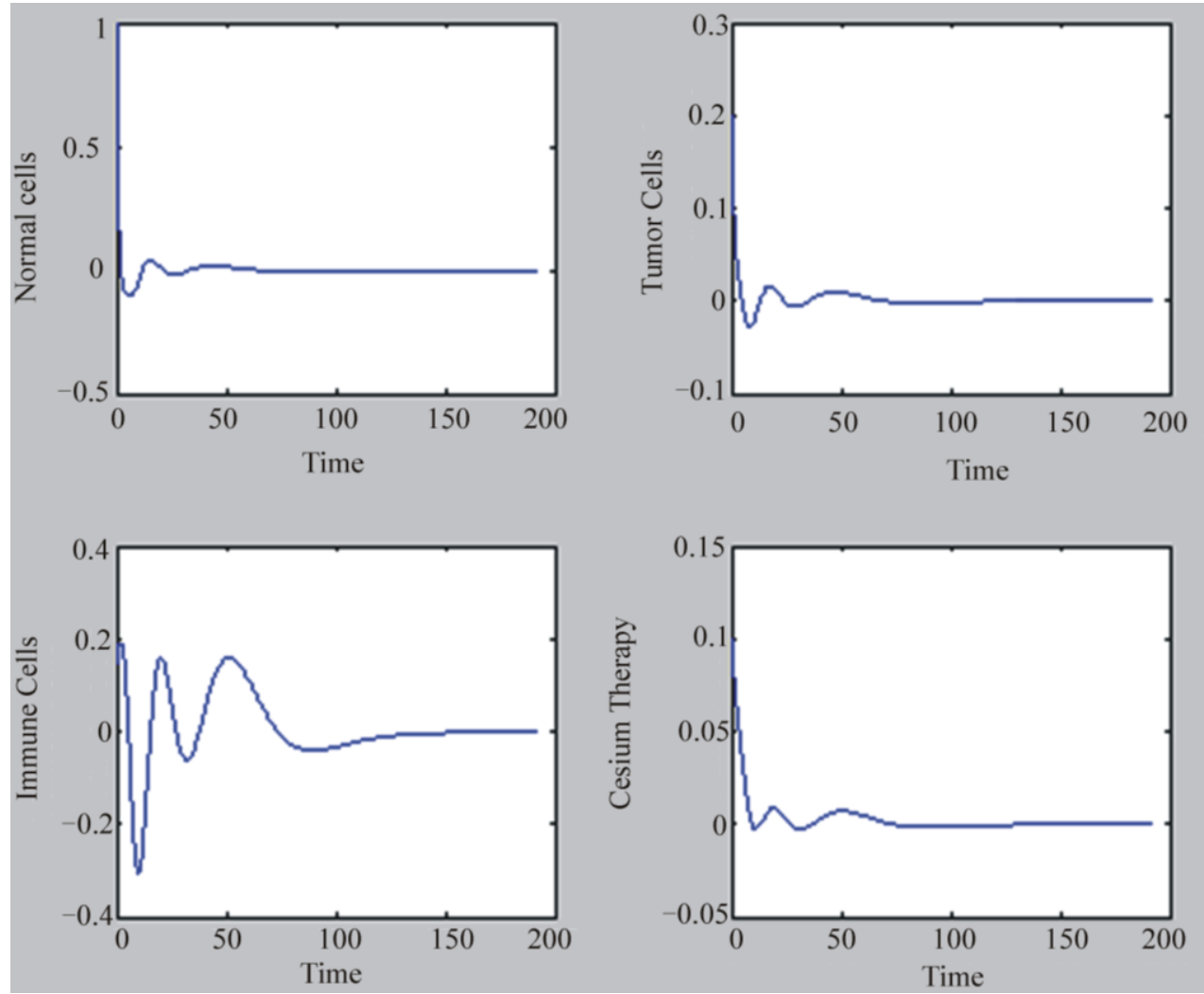

Figure 4. Normal cells, tumor cells, immune cells and therapy input response.
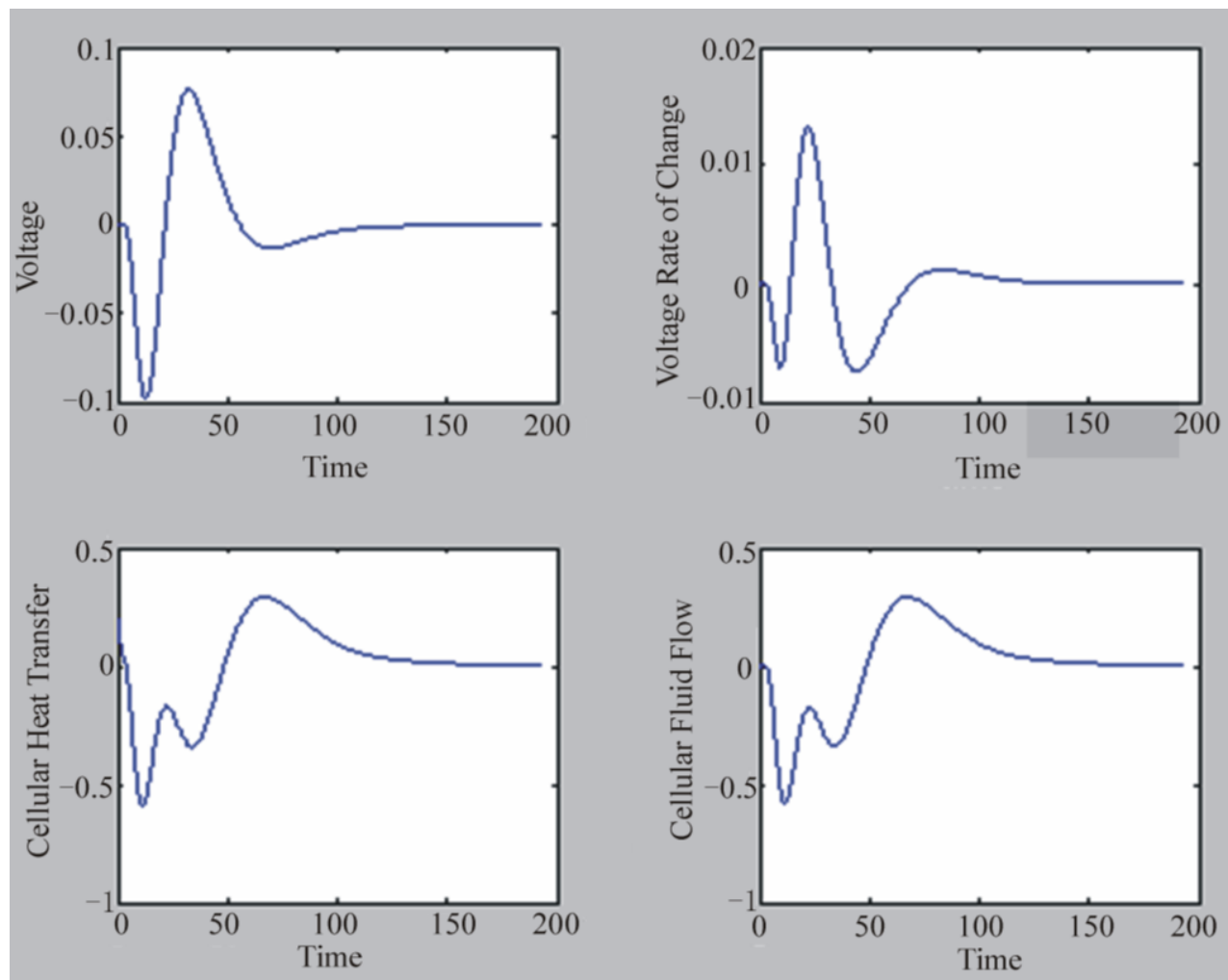

Figure 5. Cellular voltage and its rate of change, cellular heat-fluid transfer. 


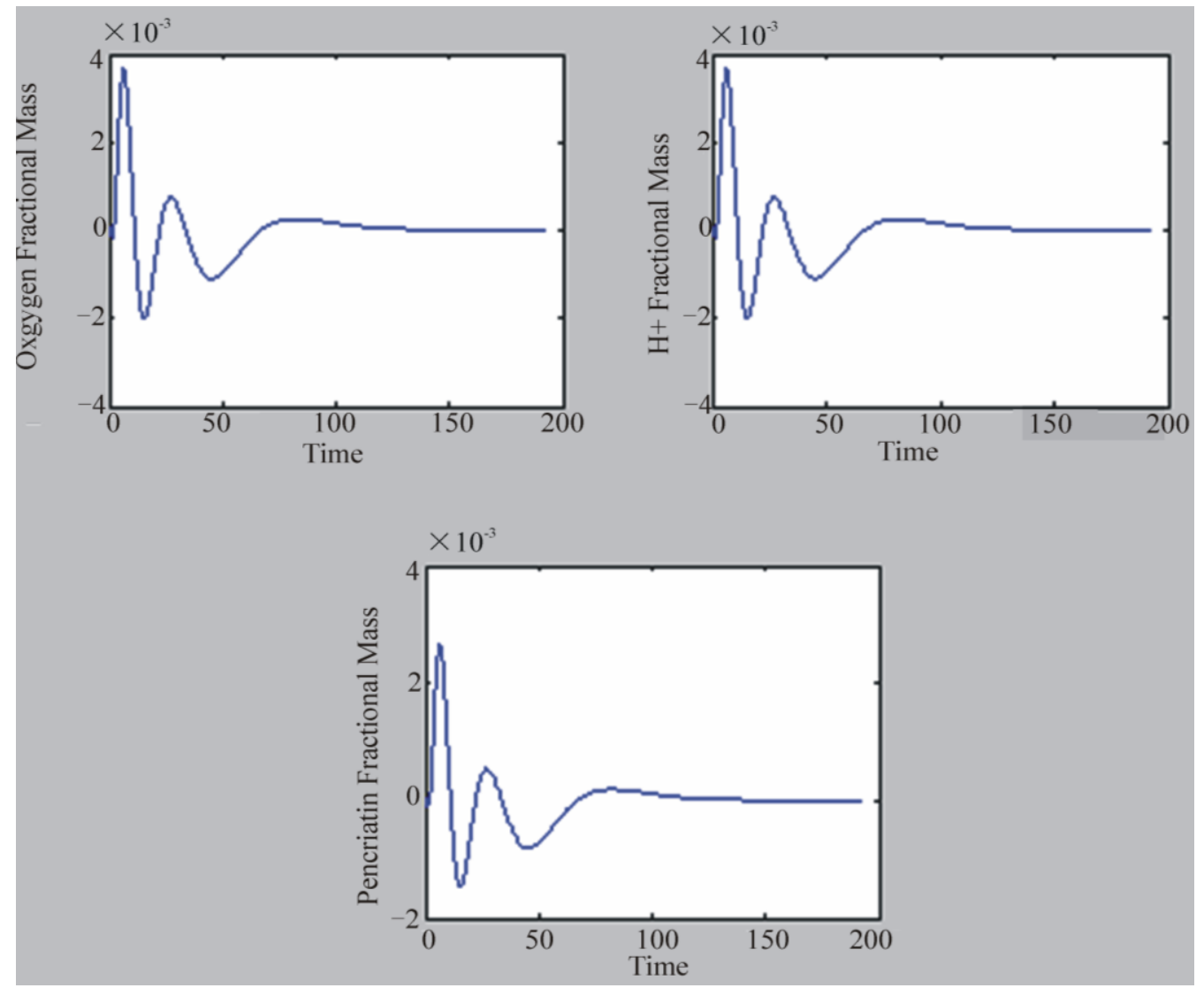

Figure 6. Fractional mass of oxygen, hydrogen ion and Pancreatin Enzyme.

by enforcing free kill cells.

Similarly, the cellular voltage is regulated at the required value. Transfer of heat and fluid from and into the cell is maintained fixed as well. Since oxygen is so significant to maintain a healthy cell and reduce or kill the tumor cells its control was so efficient to keep the normal and immune cells as desired. Acidity is controlled by the amount of hydrogen ions in the cell as well. Finally, Pancreatin ratio (which has an important role in killing tumor cells) is kept within the normal range as well.

- If $R_{i}=10^{9} \Omega$ or $R_{e}=10^{9} \Omega$ (Independently), the system is unstable.

- If both $R_{i}=R_{e}=10^{7} \Omega$, the system is unstable.

- Increasing $R_{i}$, immunity increases.

- Decreasing $R_{e}$, immunity increases significantly (20 times manifold).

- Decreasing wall capacitance $C_{W}$ increases the normal cells, tumor cells and immune cells and all others.

- Increasing of area ratio $\rho_{A}$ to 2.8 , oscillations occurs; and at 2.9 it results in unstable cells.

- Increasing oxygen ratio $\rho_{\mathrm{O}_{2}}$ to 3 will generate oscillations; and to 3.8 produces unstable cells.
- Decreasing hydrogen ratio $\rho_{H^{+}}$to 0.01 and oxygen rate $\rho_{\mathrm{O}_{2}}$ at 0.021 , generates unstable cells.

Increasing Pancreatin ratio $\rho_{P n}$ to 6 produces unstable cells.

\section{CONCLUSIONS}

This paper presents a more generalized dynamical model describing the normal-tumor-immune growth considering the electro-thermo-fluidic-chemical characteristics of a human cell. Equilibrium and stability of such a model is validated via state-feedback controller design. Cesium therapy is found to be so effective in controlling the whole system. The sensitivity and the range of the dynamic parameters that influence the normality and immunity of the live cell have been identified. It is figured out that the cellular voltage is so important to regulate the cell normal operation. Three cellular components have been investigated: oxygen (to keep the cell alive and prevent cancer growth, hydrogen (acidity: PH value) and Pancreatin enzyme (to kill the tumor cells). Simulation of the controlled therapy shows how is approach is so efficient and can be implemented clinically. 


\section{REFERENCES}

[1] Hanahan, D. and Weinberg, R.A. (2000) The hallmarks of cancer. Cell Journal, 100, 57-70. doi:10.1016/S0092-8674(00)81683-9

[2] Raines, J.K. (1981) Electromagnetic field interactions with the human body: Observed effects and theories. Technical Report, NASA, Goddard Space Flight Center, Washington.

[3] De Pillis, L.G. and Radunskaya, A.E. (2003.) The dynamics of an optimally controlled tumor model: A case study. Mathematical and Computer Modeling, 37, 12211244. doi:10.1016/S0895-7177(03)00133-X

[4] Itik M., Salamci, M.U. and Banks, S.P. (2010) SDRE optimal control of drug administration in cancer treatment. Turkish Journal of Electrical Engineering and Computer Sciences, 18, 715-729.

[5] Szymanska, Z. (2003) Analysis of immunotherapy models in the context of cancer dynamics. International Journal of Applied Mathematics and Computer Science, 13, 407-418,

[6] Byrne, H.M., Alarcon, T., Owen, R., Webb, S. D. and Maiki, P.K. (2006) Modelling aspects of cancer dynamics: A review. Philosophical Transaction of the Royal Society A, 364, 1563-1578.

[7] Kirschner, D. (2009) On the global dynamics of a model for tumor immunotherapy. Mathematical Biosciences and Engineering, 6, 573-583.

[8] U. Ledzewicz, (2005) Optimal control for a system modelling tumor anti-angiogenesis. ACSE 2005 Conference, Cairo, 19-21 December 2005.

[9] Ghaffari, A. and Nasserifar, N. (2009) Mathematical modeling and lyapunov-based drug administration in cancer chemotherapy. Journal of Electrical \& Electronic Engineering, 5, 151-158.

[10] Warburg, O.H. (1969) The prime cause and prevention of cancer. 2nd Edition, Konrad Triltsch, Würzburg.

[11] Brewer A.K. (1984) The high ph therapy for cancer, tests on mice and humans. Pharmacology Biochemistry \& Behavior, 21, 1-5.

[12] Aenold J. (2003) Clean out your arteries at home, without a needle, and at a fraction of the cost," Health Sciences Institute Members Alert, 1-4. 